\title{
Moduli Space in Homological Mirror Symmetry
}

\author{
Matsuo Sato \\ Department of Natural Science, Faculty of Education, Hirosaki University, Bunkyo-cho 1, Hirosaki, Aomori 036-8560, Japan \\ Correspondence should be addressed to Matsuo Sato; msato@hirosaki-u.ac.jp
}

Received 31 October 2018; Accepted 24 December 2018; Published 30 April 2019

Academic Editor: Dimitrios Tsimpis

Copyright (C) 2019 Matsuo Sato. This is an open access article distributed under the Creative Commons Attribution License, which permits unrestricted use, distribution, and reproduction in any medium, provided the original work is properly cited.

We prove that the moduli space of the pseudo holomorphic curves in the A-model on a symplectic torus is homeomorphic to a moduli space of Feynman diagrams in the configuration space of the morphisms in the B-model on the corresponding elliptic curve. These moduli spaces determine the $A_{\infty}$ structure of the both models.

\section{Introduction}

In the one-dimensional homological mirror symmetry (HMS) [1], the A-model on a symplectic torus corresponds to the B-model on an elliptic curve [2]. The objects, representing D-branes, are the Lagrangian submanifolds in the A-model and the complexes of the coherent sheaves in the B-model. In one dimension, any real one-dimensional submanifold is the Lagrangian and the line bundles are coherent sheaves. The morphisms, representing the open strings between the D-branes, are described by Abelian groups whose bases are given by intersecting points of the real one-dimensional submanifolds in the A-model and the maps between the complexes of the line bundles in the B-model. From these objects and morphisms, we can canonically construct the Fukaya category in the A-model and the derived category of coherent sheaves in the B-model. It is proved in [3-9] that the Fukaya category is equivalent as an $A_{\infty}$-category to the differential graded (DG) category (The DG-category is an $A_{\infty}$-category where $m_{d}(d \geq 3)$ are trivial.) canonically extended from the derived category of coherent sheaves. Furthermore, the Fukaya category is also equivalent as an $A_{\infty}$-category to a nontrivial $A_{\infty}$-category extended from the DG-category by using homotopy operators [10-18]. In this extension, $m_{3}$ is explicitly constructed in the B-model [13], whereas $m_{d}(d \geq 4)$ have not been explicitly constructed yet.

In this paper, we extend the DG-category in a different way, based on the topological string amplitudes. $m_{d}$ are newly defined and explicitly constructed in the B-model. The $A_{\infty^{-}}$ category that consists of these $m_{d}$ is shown to be equivalent to the Fukaya category (Thus, the $A_{\infty}$-category defined in this paper is equivalent as an $A_{\infty}$-category to the $A_{\infty^{-}}$ category defined by the homotopy operators because they are equivalent to the Fukaya category as $A_{\infty}$ categories.). In this construction, we find a moduli space necessary to define $m_{d}$ that satisfy the $A_{\infty}$ relations in the B-model. This moduli space is homeomorphic to the moduli space of the pseudo holomorphic curves in the A-model.

\section{Topological String Amplitudes}

The one-dimensional complex manifold in the B-model is an elliptic curve $E_{\tau}$ which is spanned by 1 and $\tau \in \mathbf{C}$. There are two ways to generalize the derived category of coherent sheaves on $E_{\tau}$ to a differential graded (DG) category. One way is based on Čech cohomology [3-9] and the other is based on Dolbeault cohomology [2, 10-16]. They are equivalent by $\breve{C}$ ech-Dolbeault isomorphism. We adopt Dolbeault cohomology. Coherent sheaves on $E_{\tau}$ are classified and constructed based on line bundles on $E_{\tau}$ [19]. We study only the line bundles. The extension to general coherent sheaves is straight forward and the way is written in $[2,13]$. The objects representing $(\mathrm{d}+1) \mathrm{D}$-branes are complexes of line bundles of degree $n_{i}, L\left(n_{i}, u_{i}\right)(i=0, \ldots, d)$, where $u_{i}$ represent connections (gauge fields) over the D-branes. The morphisms representing open strings between $L\left(n_{i-1}, u_{i-1}\right)$ and $L\left(n_{i}, u_{i}\right)$ are $H^{0, p}\left(E_{\tau}, L\left(n_{i-1, i}, u_{i-1, i}\right)\right)$, where $p$ represent the degrees of the grading $\left(p=0,1, n_{i-1, i}:=n_{i}-n_{i-1} u_{i-1, i}:=\right.$ $\left.u_{i}-u_{i-1}\right)$. 
When $n>0, H^{0,1}\left(E_{\tau}, L(n, u)\right)=0$ and the elements of $H^{0,0}\left(E_{\tau}, L(n, u)\right)$ are the basis of global sections of $L(n, u)$ :

$$
\begin{array}{r}
\theta\left[\begin{array}{l}
\frac{m}{n} \\
u
\end{array}\right](n z, n \tau) \in H^{0,0}\left(E_{\tau}, L(n, u)\right) \\
\qquad(m=0, \ldots, n-1),
\end{array}
$$

where $\theta\left[\begin{array}{l}a \\ b\end{array}\right](z, \tau)$ are theta functions with characteristics.

When $n<0, H^{0,0}\left(E_{\tau}, L(n, u)\right)=0$ and the elements of $H^{0,1}\left(E_{\tau}, L(n, u)\right)$ are the basis of harmonic $(0,1)$-forms with values in the dual bundle $L(n, u)$ :

$$
\begin{aligned}
& \overline{\theta\left[\begin{array}{c}
\frac{m}{n} \\
-u
\end{array}\right](-n z,-n \tau)} \exp \left(2 \pi t\left(n z_{2}^{2}+2 u_{2} z_{2}\right)\right) d \bar{z} \\
& \quad \in H^{0,1}\left(E_{\tau}, L(n, u)\right),
\end{aligned}
$$

where $\bar{\theta}$ is the complex conjugate of $\theta, \tau=A+i t, z=z_{1}+\tau z_{2}$, $u=u_{1}+\tau u_{2}$, and $A, t, z_{1}, z_{2}, u_{1}, u_{2} \in \mathbf{R}$.

We are concerned with the $u=0$ case for simplicity. One can easily introduce the connections. We simplify the expressions as

$$
\theta_{n}[p](z, \tau):=\theta\left[\begin{array}{l}
p \\
0
\end{array}\right](n z, n \tau)
$$

In this case, the strings are represented by

$$
\begin{aligned}
& \theta_{n}\left[\frac{m}{n}\right](z, \tau) \in H^{0,0}\left(E_{\tau}, L(n)\right) \quad(n>0), \\
& \theta_{n}^{*}\left[\frac{m}{n}\right](z, \bar{z}, \tau, \bar{\tau}) d \bar{z} \\
& \quad:=\overline{\theta_{-n}\left[\frac{m}{n}\right](z, \tau)} \exp \left(2 \pi t n z_{2}^{2}\right) d \bar{z} \\
& \in H^{0,1}\left(E_{\tau}, L(n)\right) \quad(n<0) .
\end{aligned}
$$
20]

Topological string amplitudes on $E_{\tau}$ are defined as $[9,15$,

$$
\int_{E_{\tau}} \Omega \wedge \theta_{n}^{*}\left[\frac{m}{n}\right] d \bar{z} \theta_{n_{i_{1}-1, i_{1}}}\left[\frac{m_{i_{1}-1, i_{1}}}{n_{i_{1}-1, i_{1}}}\right] \cdots \theta_{n_{i_{c}-1, i_{c}}}\left[\frac{m_{i_{c}-1, i_{c}}}{n_{i_{c}-1, i_{c}}}\right] \psi_{n_{i_{c+1}-1, i_{c+1}}}\left[\frac{m_{i_{c+1}-1, i_{c+1}}}{n_{i_{c+1}-1, i_{c+1}}}\right] \cdots \psi_{n_{i_{d}-1, i_{d}}}\left[\frac{m_{i_{d}-1, i_{d}}}{n_{i_{d}-1, i_{d}}}\right],
$$

where $n_{i_{1}-1, i_{1}}, \ldots, n_{i_{c}-1, i_{c}}>0$ and $n_{i_{c+1}-1, i_{c+1}}, \ldots, n_{i_{d}-1, i_{d}}, n<0$. $\Omega=d z$ is the holomorphic $(1,0)$-form, $\theta_{n}^{*}[m / n] d \bar{z} \epsilon$ $H^{0,1}\left(E_{\tau}, L(n)\right)$ and $\theta_{n_{i_{k}-1, i_{k}}}\left[m_{i_{k}-1, i_{k}} / n_{i_{k}-1, i_{k}}\right] \quad \in$ $H^{0,0}\left(E_{\tau}, L\left(n_{i_{k}-1, i_{k}}\right)\right)(k=1, \ldots, c)$. Here we explain what $\psi_{n}[m / n]$ is. In order to define topological string amplitudes of more than one $n<0$ states, we need to deform the theory by those states because more than one $(0,1)$-form cannot enter the topological string amplitudes in one dimension. The derived category describes such a deformed theory. In the topological string theory, the deformation by $n<0$ string states $O^{(0)} \in \Omega^{0,1}$ is given as follows. We define $O^{(1)}$ by $\widetilde{d} O^{(0)}=\left\{Q, O^{(1)}\right\}$, where $\widetilde{d}$ is a world sheet differential and $Q$ is a BRST operator. Then, the deformation of the theory is to insert $\psi:=\int_{\partial \Sigma} O^{(1)} \in \Omega^{0,0}$, where $\partial \Sigma$ is a world sheet boundary.

In our case, we define this deformation by an isomorphism $\psi: \Omega^{0,1} \longrightarrow \Omega^{0,0}$ by

$$
\begin{aligned}
O^{(0)} & =\theta_{n}^{*}\left[\frac{m}{n}\right](z, \bar{z}, \tau, \bar{\tau}) d \bar{z} \longmapsto \\
\psi & =\theta_{n}\left[\frac{m}{n}\right](z, \tau) .
\end{aligned}
$$

That is, $\theta_{n}[m / n](z, \tau) \in \Omega^{0,0}$ represent string states, when not only $n>0$ but also $n<0$. This isomorphism will be justified later by mirror symmetry of the $A_{\infty}$ structure and $m_{d}$. By using this isomorphism, (5) is written as

$$
\begin{gathered}
\int_{E_{\tau}} \Omega \wedge \theta_{n}^{*}\left[\frac{m}{n}\right] d \bar{z} \theta_{n_{0,1}}\left[\frac{m_{0,1}}{n_{0,1}}\right] \\
\cdot \theta_{n_{1,2}}\left[\frac{m_{1,2}}{n_{1,2}}\right] \cdots \theta_{n_{d-1, d}}\left[\frac{m_{d-1, d}}{n_{d-1, d}}\right] .
\end{gathered}
$$

On the other hand, (5) should also be written by using $m_{d}$ in $A_{\infty}$-category $[15,20]$ like

$$
\sim \int_{E_{\tau}} \Omega \wedge \theta_{n}^{*}\left[\frac{m}{n}\right] d \bar{z} m_{d}\left(\frac{m_{0,1}}{n_{0,1}}, \ldots, \frac{m_{d-1, d}}{n_{d-1, d}}\right) .
$$

Therefore, $m_{d}$ should be defined like

$$
\begin{aligned}
m_{d} & \left(\frac{m_{0,1}}{n_{0,1}}, \ldots, \frac{m_{d-1, d}}{n_{d-1, d}}\right) \\
& \sim \theta_{n_{0,1}}\left[\frac{m_{0,1}}{n_{0,1}}\right] \cdots \theta_{n_{d-1, d}}\left[\frac{m_{d-1, d}}{n_{d-1, d}}\right] .
\end{aligned}
$$

We will define completely $m_{d}$ that possesses $A_{\infty}$ structure in the next section.

Here we discuss consistency of the integration over $E_{\tau}$ with the periodicity. Whereas the theta functions are invariant under $z \longmapsto z+1$, they are transformed under $z \longmapsto z+\tau$ as

$$
\begin{gathered}
\theta_{n}\left[\frac{m}{n}\right](z, \tau) \longmapsto e^{-\pi i n(2 z+\tau)} \theta_{n}\left[\frac{m}{n}\right](z, \tau) \\
\theta_{n}^{*}\left[\frac{m}{n}\right](z, \bar{z}, \tau, \bar{\tau}) d \bar{z} \\
\longmapsto e^{-\pi i n(2 z+\tau)} \theta_{n}^{*}\left[\frac{m}{n}\right](z, \bar{z}, \tau, \bar{\tau}) d \bar{z}
\end{gathered}
$$


Then, the integrand is transformed as

$$
\theta_{n}^{*}\left[\frac{m}{n}\right] d \bar{z} \theta_{n_{0,1}}\left[\frac{m_{0,1}}{n_{0,1}}\right] \cdots \theta_{n_{d-1, d}}\left[\frac{m_{d-1, d}}{n_{d-1, d}}\right] \longmapsto e^{-\pi i(2 z+\tau)\left(n+n_{0,1}+\cdots+n_{d-1, d}\right)} \theta_{n}^{*}\left[\frac{m}{n}\right] d \bar{z} \theta_{n_{0,1}}\left[\frac{m_{0,1}}{n_{0,1}}\right] \cdots \theta_{n_{d-1, d}}\left[\frac{m_{d-1, d}}{n_{d-1, d}}\right]
$$

Because this should be invariant for periodicity, $n$ needs to be $-\left(n_{0,1}+\cdots+n_{d-1, d}\right)$ and then $n_{0,1}+\cdots+n_{d-1, d}>0$.

\section{3. $m_{d}$ and $A_{\infty}$ Structure}

In order to define $m_{d}$, we multiply theta functions with characteristics. They are defined by series as follows.

Definition 1 (theta functions with characteristics).

$$
\begin{aligned}
\theta\left[\begin{array}{l}
a \\
b
\end{array}\right](z, \tau) \\
\quad:=\sum_{m \in \mathbf{Z}} \exp \left(\pi i(m+a)^{2} \tau+2 \pi i(m+a)(z+b)\right),
\end{aligned}
$$

where $a \in \mathbf{R} / \mathbf{Z}, b \in \mathbf{C}$.

A product formula is given by the following.

\section{Theorem 2.}

$$
\begin{aligned}
& \theta\left[\begin{array}{c}
\frac{a_{1}}{n_{1}} \\
0
\end{array}\right]\left(z_{1}, n_{1} \tau\right) \theta\left[\begin{array}{c}
\frac{a_{2}}{n_{2}} \\
0
\end{array}\right]\left(z_{2}, n_{2} \tau\right) \\
& \quad=\sum_{d \in \mathbf{Z} /\left(n_{1}+n_{2}\right) \mathbf{Z}} \theta\left[\begin{array}{c}
\frac{n_{2} a_{1}-n_{1} a_{2}+n_{1} n_{2} d}{n_{1} n_{2}\left(n_{1}+n_{2}\right)} \\
0
\end{array}\right]\left(n_{2} z_{1}\right. \\
& -n_{1} z_{2}, n_{1} n_{2}\left(n_{1}+n_{2}\right) \\
& \cdot \tau) \theta\left[\frac{\frac{a_{1}+a_{2}+n_{1} d}{n_{1}+n_{2}}}{0}\right]\left(z_{1}+z_{2},\left(n_{1}+n_{2}\right) \tau\right)
\end{aligned}
$$

where $n_{1}, n_{2} \in \mathbf{Z}, a_{1}, a_{2} \in \mathbf{R}$, and $z_{1}, z_{2}, \tau \in \mathbf{C}$.

While this formula was proved as an addition formula when $n_{1}, n_{2} \in \mathbf{N}$ in [21], it can also be proved as series when $n_{1}, n_{2} \in \mathbf{Z}$ as follows.

Proof.

$$
\begin{aligned}
& \text { (1.h.s.) }=\sum_{m_{1}, m_{2} \in \mathbf{Z}} \exp \left(\pi i n_{1} \tau\left(m_{1}+\frac{a_{1}}{n_{1}}\right)^{2}+2 \pi i\left(m_{1}\right.\right. \\
& \left.+\frac{a_{1}}{n_{1}}\right) z_{1}+\pi i n_{2} \tau\left(m_{2}+\frac{a_{2}}{n_{2}}\right)^{2} \\
& \left.+2 \pi i\left(m_{2}+\frac{a_{2}}{n_{2}}\right) z_{2}\right)
\end{aligned}
$$

$$
\begin{aligned}
= & \sum_{l, m \in \mathbf{Z}, d \in \mathbf{Z} /\left(n_{1}+n_{2}\right) \mathbf{Z}} \exp \pi i\left(\left(n_{1}+n_{2}\right) \tau l^{2}\right. \\
& +2\left(\left(a_{1}+a_{2}\right) \tau+z_{1}+z_{2}\right) l+2 n_{1} \tau l d+n_{1} \tau d^{2} \\
& +\left(2 a_{1} \tau+2 z_{1}\right) d+n_{1} \tau\left(n_{1}+n_{2}\right)^{2} m^{2} \\
& +2 n_{1}\left(n_{1}+n_{2}\right) \tau l m \\
+ & 2\left(a_{1} \tau+z_{1}\right)\left(n_{1}+n_{2}\right) m+2 n_{1}\left(n_{1}+n_{2}\right) \tau m d+\frac{a_{1}^{2}}{n_{1}} \tau \\
& \left.+\frac{a_{2}^{2}}{n_{2}} \tau+2 \frac{a_{1}}{n_{1}} z_{1}+2 \frac{a_{2}}{n_{2}} z_{2}\right),
\end{aligned}
$$

where we have set $m_{1}=l+p, m_{2}=l(l, p \in \mathbf{Z})$, and $p=$ $\left(n_{1}+n_{2}\right) m+d\left(m \in \mathbf{Z}, d \in \mathbf{Z} /\left(n_{1}+n_{2}\right) \mathbf{Z}\right)$. On the other hand,

$$
\begin{aligned}
& \text { (r.h.s. }=\sum_{m, n \in \mathbf{Z}, d \in \mathbf{Z} /\left(n_{1}+n_{2}\right) \mathbf{Z}} \exp \left(\pi i n_{1} n_{2}\left(n_{1}+n_{2}\right)\right. \\
& \cdot \tau\left(m+\frac{n_{2} a_{1}-n_{1} a_{2}+n_{1} n_{2} d}{n_{1} n_{2}\left(n_{1}+n_{2}\right)}\right)^{2} \\
& \left.+2 \pi i\left(m+\frac{n_{2} a_{1}-n_{1} a_{2}+n_{1} n_{2} d}{n_{1} n_{2}\left(n_{1}+n_{2}\right)}\right)\left(n_{2} z_{1}-n_{1} z_{2}\right)\right) \\
& ++\pi i\left(n_{1}+n_{2}\right) \tau\left(n+\frac{a_{1}+a_{2}+n_{1} d}{n_{1}+n_{2}}\right)^{2} \\
& \left.+2 \pi i\left(n+\frac{a_{1}+a_{2}+n_{1} d}{n_{1}+n_{2}}\right)\left(z_{1}+z_{2}\right)\right) .
\end{aligned}
$$

If we set $n=l+n_{1} m(l \in \mathbf{Z})$, (15) coincides with (14).

In a special case, $z_{1}=n_{1} z+u_{1}$ and $z_{2}=n_{2} z+u_{2}\left(u_{1}, u_{2} \epsilon\right.$ C), we obtain

$$
\begin{aligned}
& \theta\left[\begin{array}{l}
\frac{a_{1}}{n_{1}} \\
u_{1}
\end{array}\right]\left(n_{1} z, n_{1} \tau\right) \theta\left[\begin{array}{l}
\frac{a_{2}}{n_{2}} \\
u_{2}
\end{array}\right]\left(n_{2} z, n_{2} \tau\right) \\
& \quad=\sum_{d \in \mathbf{Z} /\left(n_{1}+n_{2}\right) \mathbf{Z}} \theta\left[\begin{array}{c}
\frac{n_{2} a_{1}-n_{1} a_{2}+n_{1} n_{2} d}{n_{1} n_{2}\left(n_{1}+n_{2}\right)} \\
n_{2} u_{1}-n_{1} u_{2}
\end{array}\right] \\
& \cdot\left(0, n_{1} n_{2}\left(n_{1}+n_{2}\right)\right. \\
& \cdot \tau) \theta\left[\begin{array}{c}
\frac{a_{1}+a_{2}+n_{1} d}{n_{1}+n_{2}} \\
u_{1}+u_{2}
\end{array}\right]\left(\left(n_{1}+n_{2}\right) z,\left(n_{1}+n_{2}\right) \tau\right) .
\end{aligned}
$$


This product is expanded by the theta functions with complex coefficients. The coefficients are independent of $z$. We simplify this formula. Because $\left(a_{1}+a_{2}+n_{1} d\right) /\left(n_{1}+n_{2}\right) \in \mathbf{R} / \mathbf{Z}$ on the last line, we can add $-\left(\left(n_{1}+n_{2}\right) /\left(n_{1}+n_{2}\right)\right) d$ and obtain

$$
\begin{aligned}
& \theta\left[\begin{array}{l}
\frac{a_{1}}{n_{1}} \\
u_{1}
\end{array}\right]\left(n_{1} z, n_{1} \tau\right) \theta\left[\begin{array}{l}
\frac{a_{2}}{n_{2}} \\
u_{2}
\end{array}\right]\left(n_{2} z, n_{2} \tau\right) \\
& =\sum_{d \in \mathbf{Z} /\left(n_{1}+n_{2}\right) \mathbf{Z}} \theta\left[\begin{array}{c}
\frac{n_{2} a_{1}-n_{1}\left(a_{2}-n_{2} d\right)}{n_{1} n_{2}\left(n_{1}+n_{2}\right)} \\
n_{2} u_{1}-n_{1} u_{2}
\end{array}\right]
\end{aligned}
$$

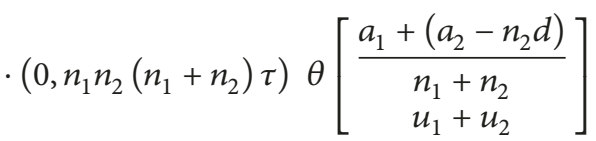

$$
\begin{aligned}
& \cdot\left(\left(n_{1}+n_{2}\right) z,\left(n_{1}+n_{2}\right) \tau\right) \\
& =\sum_{m \in \mathbf{Z}, d \in \mathbf{Z} /\left(n_{1}+n_{2}\right) \mathbf{Z}} \exp \left(\pi i n_{1} n_{2}\left(n_{1}+n_{2}\right) \tau\left(m+\frac{n_{2} a_{1}-n_{1}\left(a_{2}-n_{2} d\right)}{n_{1} n_{2}\left(n_{1}+n_{2}\right)}\right)^{2}\right. \\
& \left.+2 \pi i\left(m+\frac{n_{2} a_{1}-n_{1}\left(a_{2}-n_{2} d\right)}{n_{1} n_{2}\left(n_{1}+n_{2}\right)}\right)\left(n_{2} u_{1}-n_{1} u_{2}\right)\right) \\
& \cdot \theta\left[\begin{array}{c}
\frac{a_{1}+\left(a_{2}-n_{2} d\right)}{n_{1}+n_{2}} \\
u_{1}+u_{2}
\end{array}\right]\left(\left(n_{1}+n_{2}\right) z,\left(n_{1}+n_{2}\right) \tau\right) \text {. }
\end{aligned}
$$

Because $\left(a_{1}+\left(a_{2}-n_{2} d\right)\right) /\left(n_{1}+n_{2}\right) \in \mathbf{R} / \mathbf{Z}$ on the last line, we can add $-\left(n_{2}\left(n_{1}+n_{2}\right) m /\left(n_{1}+n_{2}\right)\right) d$. By defining $\alpha:=\left(n_{1}+\right.$ $\left.n_{2}\right) m+d(\alpha \in \mathbf{Z})$, we obtain

\section{Lemma 3.}

$$
\begin{aligned}
& \theta\left[\begin{array}{l}
\frac{a_{1}}{n_{1}} \\
u_{1}
\end{array}\right]\left(n_{1} z, n_{1} \tau\right) \theta\left[\begin{array}{l}
\frac{a_{2}}{n_{2}} \\
u_{2}
\end{array}\right]\left(n_{2} z, n_{2} \tau\right) \\
& =\sum_{\alpha \in \mathbf{Z}} \exp \left(\pi i \tau \frac{\left(n_{2} a_{1}-n_{1}\left(a_{2}-n_{2} \alpha\right)\right)^{2}}{n_{1} n_{2}\left(n_{1}+n_{2}\right)}\right. \\
& \left.+2 \pi i\left(\frac{n_{2} a_{1}-n_{1}\left(a_{2}-n_{2} \alpha\right)}{n_{1} n_{2}\left(n_{1}+n_{2}\right)}\right)\left(n_{2} u_{1}-n_{1} u_{2}\right)\right) \\
& \cdot \theta\left[\begin{array}{c}
\frac{a_{1}+\left(a_{2}-n_{2} \alpha\right)}{n_{1}+n_{2}} \\
u_{1}+u_{2}
\end{array}\right]\left(\left(n_{1}+n_{2}\right) z,\left(n_{1}+n_{2}\right) \tau\right) .
\end{aligned}
$$

Especially, when $u=0$ we obtain

$$
\begin{aligned}
\theta_{n_{1}} & {\left[\frac{a_{1}}{n_{1}}\right](z, \tau) \theta_{n_{2}}\left[\frac{a_{2}}{n_{2}}\right](z, \tau) } \\
= & \sum_{\alpha \in \mathbf{Z}} \exp \left(\pi i \tau \frac{\left(n_{2} a_{1}-n_{1}\left(a_{2}-n_{2} \alpha\right)\right)^{2}}{n_{1} n_{2}\left(n_{1}+n_{2}\right)}\right) \\
\cdot & \theta_{n_{1}+n_{2}}\left[\frac{a_{1}+\left(a_{2}-n_{2} \alpha\right)}{n_{1}+n_{2}}\right](z, \tau) .
\end{aligned}
$$

From now on, we abbreviate $\theta_{n}[p](z, \tau)$ to $\theta_{n}[p]$ because $(z, \tau)$ does not vary. By using this formula, we obtain the following.

\section{Theorem 4.}

$$
\begin{aligned}
\theta_{n_{0,1}} & {\left[\frac{a_{0,1}}{n_{0,1}}\right] \theta_{n_{1,2}}\left[\frac{a_{1,2}}{n_{1,2}}\right] \cdots \theta_{n_{d-1, d}}\left[\frac{a_{d-1, d}}{n_{d-1, d}}\right] } \\
= & \sum_{\alpha_{1,2}, \ldots, \alpha_{d-1, d} \in \mathbf{Z}} \exp \left(2 \pi i \tau S_{d}\left(\frac{\widetilde{a}_{0,1}}{n_{0,1}}, \ldots, \frac{\widetilde{a}_{d-1, d}}{n_{d-1, d}}\right)\right) \\
\cdot \theta_{n_{0,1}+\cdots+n_{d-1, d}} & {\left[\frac{\tilde{a}_{0,1}+\cdots+\tilde{a}_{d-1, d}}{n_{0,1}+\cdots+n_{d-1, d}}\right], }
\end{aligned}
$$

where

$$
\begin{aligned}
& \widetilde{a}_{i-1, i}:=a_{i-1, i}-n_{i-1, i} \alpha_{i-1, i} \quad(i=1, \ldots, d) \\
& \alpha_{0,1} \equiv 0 \\
& S_{d}\left(p_{0,1}, \ldots, p_{d-1, d}\right) \\
& \quad:=\frac{1}{2}\left(\left(\sum_{i=1}^{d} n_{i-1, i} p_{i-1, i}\left(p_{i-1, i}-1\right)\right)\right. \\
& \left.\quad-n_{0, d} p_{0, d}\left(p_{0, d}-1\right)\right)
\end{aligned}
$$




$$
\begin{aligned}
& n_{0, d}:=n_{0,1}+\cdots+n_{d-1, d} \\
& p_{0, d}:=\frac{1}{n_{0, d}} \sum_{i=1}^{d} n_{i-1, i} p_{i-1, i} .
\end{aligned}
$$

Proof. We start with $d=2$ case. Explicitly,

$$
S_{2}\left(\frac{\tilde{a}_{0,1}}{n_{0,1}}, \frac{\tilde{a}_{1,2}}{n_{1,2}}\right)=\frac{\left(n_{1,2} \tilde{a}_{0,1}-n_{0,1} \tilde{a}_{1,2}\right)^{2}}{2 n_{0,1} n_{1,2}\left(n_{0,1}+n_{1,2}\right)} .
$$

Then, Lemma 3 implies Theorem 4 is right when $d=2$. For $2 \leq k \leq d-1$, explicitly,

$$
\begin{aligned}
& S_{k+1}\left(\frac{\widetilde{a}_{0,1}}{n_{0,1}}, \ldots, \frac{\widetilde{a}_{k, k+1}}{n_{k, k+1}}\right)-S_{k}\left(\frac{\widetilde{a}_{0,1}}{n_{0,1}}, \ldots, \frac{\widetilde{a}_{k-1, k}}{n_{k-1, k}}\right) \\
& =\frac{\left(n_{k, k+1}\left(\widetilde{a}_{0,1}+\cdots+\widetilde{a}_{k-1, k}\right)-\left(n_{0,1}+\cdots+n_{k-1, k}\right) \widetilde{a}_{k, k+1}\right)^{2}}{2\left(n_{0,1}+\cdots+n_{k-1, k}\right) n_{k, k+1}\left(n_{0,1}+\cdots+n_{k-1, k}+n_{k, k+1}\right)} .
\end{aligned}
$$

Then, Lemma 3 implies

$$
\begin{aligned}
& \theta_{n_{0,1}+\cdots+n_{k-1, k}}\left[\frac{\widetilde{a}_{0,1}+\cdots+\widetilde{a}_{k-1, k}}{n_{0,1}+\cdots+n_{k-1, k}}\right] \theta_{n_{k, k+1}}\left[\frac{a_{k, k+1}}{n_{k, k+1}}\right] \\
& \quad=\sum_{\alpha_{k+1} \in \mathbf{Z}} \exp \left(2 \pi i \tau\left(S_{k+1}\left(\frac{\widetilde{a}_{0,1}}{n_{0,1}}, \ldots, \frac{\widetilde{a}_{k, k+1}}{n_{k, k+1}}\right)-S_{k}\left(\frac{\widetilde{a}_{0,1}}{n_{0,1}}, \ldots, \frac{\widetilde{a}_{k-1, k}}{n_{k-1, k}}\right)\right)\right) \\
& \cdot \theta_{n_{0,1}+\cdots+n_{k, k+1}}\left[\frac{\widetilde{a}_{0,1}+\cdots+\widetilde{a}_{k, k+1}}{n_{0,1}+\cdots+n_{k, k+1}}\right] .
\end{aligned}
$$

Therefore,

$$
\begin{gathered}
\theta_{n_{0,1}}\left[\frac{a_{0,1}}{n_{0,1}}\right] \theta_{n_{1,2}}\left[\frac{a_{1,2}}{n_{1,2}}\right] \cdots \theta_{n_{d-1, d}}\left[\frac{a_{d-1, d}}{n_{d-1, d}}\right] \\
=\sum_{\alpha_{1,2} \in \mathbf{Z}} \exp \left(2 \pi i \tau S_{2}\left(\frac{\tilde{a}_{0,1}}{n_{0,1}}, \frac{\tilde{a}_{1,2}}{n_{1,2}}\right)\right) \\
\cdot \theta_{n_{0,1}+n_{1,2}}\left[\frac{\tilde{a}_{0,1}+\tilde{a}_{1,2}}{n_{0,1}+n_{1,2}}\right] \\
\cdot \theta_{n_{2,3}}\left[\frac{a_{2,3}}{n_{2,3}}\right] \cdots \theta_{n_{d-1, d}}\left[\frac{a_{d-1, d}}{n_{d-1, d}}\right] \\
=\sum_{\alpha_{1,2} \in \mathbf{Z}} \exp \left(2 \pi i \tau S_{2}\left(\frac{\tilde{a}_{0,1}}{n_{0,1}}, \frac{\tilde{a}_{1,2}}{n_{1,2}}\right)\right) \\
\cdot \sum_{\alpha_{2,3} \in \mathbf{Z}} \exp \left(2 \pi i \tau \left(S_{3}\left(\frac{\tilde{a}_{0,1}}{n_{0,1}}, \frac{\tilde{a}_{1,2}}{n_{1,2}}, \frac{\tilde{a}_{2,3}}{n_{2,3}}\right)\right.\right. \\
\left.\left.-S_{2}\left(\frac{\tilde{a}_{0,1}}{n_{0,1}}, \frac{\tilde{a}_{1,2}}{n_{1,2}}\right)\right)\right)
\end{gathered}
$$

$$
\begin{aligned}
& \cdot \theta_{n_{0,1}+n_{1,2}+n_{2,3}}\left[\frac{\tilde{a}_{0,1}+\tilde{a}_{1,2}+\tilde{a}_{2,3}}{n_{0,1}+n_{1,2}+n_{2,3}}\right] \theta_{n_{3,4}}\left[\frac{a_{3,4}}{n_{3,4}}\right] \\
& \cdots \theta_{n_{d-1, d}}\left[\frac{a_{d-1, d}}{n_{d-1, d}}\right] \\
& =\sum_{\alpha_{1,2}, \alpha_{2,3} \in \mathbf{Z}} \exp \left(2 \pi i \tau S_{3}\left(\frac{\tilde{a}_{0,1}}{n_{0,1}}, \frac{\tilde{a}_{1,2}}{n_{1,2}}, \frac{\tilde{a}_{2,3}}{n_{2,3}}\right)\right) \\
& \cdot \theta_{n_{0,1}+n_{1,2}+n_{2,3}}\left[\frac{\tilde{a}_{0,1}+\tilde{a}_{1,2}+\tilde{a}_{2,3}}{n_{0,1}+n_{1,2}+n_{2,3}}\right] \theta_{n_{3,4}}\left[\frac{a_{3,4}}{n_{3,4}}\right] \\
& \cdots \theta_{n_{d-1, d}}\left[\frac{a_{d-1, d}}{n_{d-1, d}}\right]=\cdots \\
& =\sum_{\alpha_{1,2}, \ldots, \alpha_{d-1, d} \in \mathbf{Z}} \exp \left(2 \pi i \tau S_{d}\left(\frac{\tilde{a}_{0,1}}{n_{0,1}}, \ldots, \frac{\tilde{a}_{d-1, d}}{n_{d-1, d}}\right)\right) \\
& \cdot \theta_{n_{0,1}+\cdots+n_{d-1, d}}\left[\frac{\widetilde{a}_{0,1}+\cdots+\widetilde{a}_{d-1, d}}{n_{0,1}+\cdots+n_{d-1, d}}\right] \text {. }
\end{aligned}
$$

Next, we study $A_{\infty}$ structure. We define an extended theta function $\widetilde{\theta}_{n}[\tilde{p}]$ whose configuration space is a universal cover $\mathbf{R}(\ni \tilde{p})$ of the configuration space $\mathbf{R} / \mathbf{Z}(\ni p)$ of the theta function $\theta_{n}[p]$. That is, $\widetilde{\theta}_{n}[a / n+1] \neq \widetilde{\theta}_{n}[a / n]$ whereas $\theta_{n}[a / n+$ $1]=\theta_{n}[a / n]$. The product is defined as follows.

Definition 5 (product of $\widetilde{\theta})$.

$$
\begin{gathered}
\widetilde{\theta}_{n_{0,1}}\left[\frac{a_{0,1}}{n_{0,1}}\right](z, \tau) \widetilde{\theta}_{n_{1,2}}\left[\frac{a_{1,2}}{n_{1,2}}\right](z, \tau) \\
:=\exp \left(\pi i \tau \frac{\left(n_{1,2} a_{0,1}-n_{0,1} a_{1,2}\right)^{2}}{n_{0,1} n_{1,2}\left(n_{0,1}+n_{1,2}\right)}\right) \\
\cdot \widetilde{\theta}_{n_{0,1}+n_{1,2}}\left[\frac{a_{0,1}+a_{1,2}}{n_{0,1}+n_{1,2}}\right](z, \tau) .
\end{gathered}
$$

This definition leads to the following.

\section{Lemma 6.}

$$
\begin{gathered}
\sum_{\alpha \in \mathbf{Z}} \tilde{\theta}_{n_{0,1}}\left[\frac{a_{0,1}}{n_{0,1}}\right](z, \tau) \tilde{\theta}_{n_{1,2}}\left[\frac{a_{1,2}}{n_{1,2}}-\alpha\right](z, \tau) \\
=\sum_{\alpha \in \mathbf{Z}} \exp \left(\pi i \tau \frac{\left(n_{1,2} a_{0,1}-n_{0,1}\left(a_{1,2}-n_{1,2} \alpha\right)\right)^{2}}{n_{0,1} n_{1,2}\left(n_{0,1}+n_{1,2}\right)}\right) \\
\cdot \widetilde{\theta}_{n_{0,1}+n_{1,2}}\left[\frac{a_{0,1}+\left(a_{1,2}-n_{1,2} \alpha\right)}{n_{0,1}+n_{1,2}}\right](z, \tau) .
\end{gathered}
$$

Proof. Clear. 


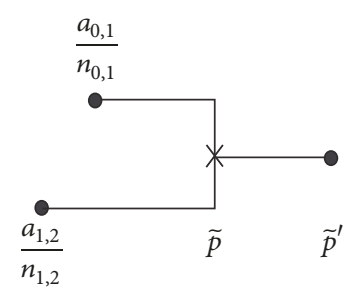

FIGURE 1: Three-point interaction.

The coefficients in this formula coincide with those of Lemma 3. Furthermore, the definition leads to the following.

Lemma 7 (local product in the configuration space).

$$
\tilde{\theta}_{n_{0,1}}[\tilde{p}](z, \tau) \tilde{\theta}_{n_{1,2}}[\tilde{p}](z, \tau)=\tilde{\theta}_{n_{0,1}+n_{1,2}}[\tilde{p}](z, \tau)
$$

Proof.

$$
\begin{gathered}
\text { (1.h.s.) }=\exp \left(\pi i \tau \frac{\left(n_{1,2} n_{0,1} \tilde{p}-n_{0,1} n_{1,2} \widetilde{p}\right)^{2}}{n_{0,1} n_{1,2}\left(n_{0,1}+n_{1,2}\right)}\right) \\
\cdot \tilde{\theta}_{n_{0,1}+n_{1,2}}\left[\frac{n_{0,1} \tilde{p}+n_{1,2} \tilde{p}}{n_{0,1}+n_{1,2}}\right](z, \tau)=\text { (r.h.s.). }
\end{gathered}
$$

Next, we define a propagator $\widetilde{G}_{n}\left[\widetilde{p}_{0}, \widetilde{p}\right](z, \tau)$ in the configuration space as follows.

Definition 8 (propagator in the configuration space).

$$
\widetilde{G}_{n}\left[\tilde{p}_{0}, \widetilde{p}\right](z, \tau) \tilde{\theta}_{n}[\tilde{p}](z, \tau):=\tilde{\theta}_{n}\left[\tilde{p}_{0}\right](z, \tau) .
$$

As a result, Feynman diagrams appear in the configuration space (Figure 1):

$$
\begin{aligned}
& \widetilde{\theta}_{n_{0,1}}\left[\frac{a_{0,1}}{n_{0,1}}\right] \widetilde{\theta}_{n_{1,2}}\left[\frac{a_{1,2}}{n_{1,2}}\right]=\widetilde{G}_{n_{0,1}}\left[\frac{a_{0,1}}{n_{0,1}}, \widetilde{p}\right] \\
& \cdot \widetilde{G}_{n_{1,2}}\left[\frac{a_{1,2}}{n_{1,2}}, \widetilde{p}\right] \widetilde{\theta}_{n_{0,1}}[\widetilde{p}] \widetilde{\theta}_{n_{1,2}}[\tilde{p}] \\
& =\widetilde{G}_{n_{0,1}}\left[\frac{a_{0,1}}{n_{0,1}}, \widetilde{p}\right] \widetilde{G}_{n_{1,2}}\left[\frac{a_{1,2}}{n_{1,2}}, \widetilde{p}\right] \widetilde{\theta}_{n_{0,1}+n_{1,2}}[\tilde{p}] . \\
& =\widetilde{G}_{n_{0,1}}\left[\frac{a_{0,1}}{n_{0,1}}, \widetilde{p}\right] \widetilde{G}_{n_{1,2}}\left[\frac{a_{1,2}}{n_{1,2}}, \widetilde{p}\right] \widetilde{G}_{n_{0,1}+n_{1,2}}\left[\tilde{p}, \widetilde{p}^{\prime}\right] \\
& \cdot \widetilde{\theta}_{n_{0,1}+n_{1,2}}\left[\widetilde{p}^{\prime}\right] .
\end{aligned}
$$

When external states $\tilde{\theta}_{n}[m / n]$ propagate from $m / n$ to $\tilde{p}$, we can parametrize $\widetilde{p}=(m+v) / n$. If two external states $\widetilde{\theta}_{n_{0,1}}\left[m_{0,1} / n_{0,1}\right]$ and $\widetilde{\theta}_{n_{1,2}}\left[m_{1,2} / n_{1,2}\right]$ propagate to the same point $\tilde{p}=\left(m_{0,1}+v_{0,1}\right) / n_{0,1}=\left(m_{1,2}+v_{1,2}\right) / n_{1,2}$ and interact locally, Lemma 7 leads to

$$
\begin{gathered}
\tilde{p}_{0,1} \quad \tilde{p}_{1,2} \\
\text { Figure 2: } \tilde{p}_{0,1} . \\
\widetilde{\theta}_{n_{0,1}}\left[\frac{m_{0,1}+v_{0,1}}{n_{0,1}}\right] \widetilde{\theta}_{n_{1,2}}\left[\frac{m_{1,2}+v_{1,2}}{n_{1,2}}\right] \\
=\widetilde{\theta}_{n_{0,1}+n_{1,2}}\left[\frac{m_{0,1}+m_{1,2}+v_{0,1}+v_{1,2}}{n_{0,1}+n_{1,2}}\right],
\end{gathered}
$$

because $\tilde{p}=\left(m_{0,1}+m_{1,2}+v_{0,1}+v_{1,2}\right) /\left(n_{0,1}+n_{1,2}\right)$. That is, $n$, $m$, and $v$ are preserved. Then, we define a canonical form of $\widetilde{\theta}$, including internal states, as $\widetilde{\theta}_{n}[(m+v) / n]$, where $n, m \in \mathbf{Z}$ and $v \in \mathbf{R}$. As a result, $m$ represent a kind of preserved numbers of string states. $\Delta \widetilde{p}=\Delta v / n=\left(v^{\prime}-v\right) / n$ represents how long the state propagates in an expression $\widetilde{G}_{n}\left[(m+v) / n,\left(m+v^{\prime}\right) / n\right]$.

We define the direction of the propagator $\widetilde{G}_{n}[(m+$ $\left.v) / n,\left(m+v^{\prime}\right) / n\right]$ as the same as the direction of $v$ and $v^{\prime}$ ( $v$ and $v^{\prime}$ should have the same direction.). Because incoming states propagate from $m / n$ to $(m+v) / n, \Delta \widetilde{p}=(m+v) / n-m / n=v / n$. Coincidence of the signs of $\Delta \widetilde{p}$ and $v$ implies that $n>0$ or $\Delta \widetilde{p}=0$. That is, incoming states with $n<0$ cannot propagate. Similarly, outgoing states with $n>0$ cannot propagate.

Strings between (i-1)-th and i-th D-branes can interact only with strings between (i-2)-th and (i-1)-th D-branes and strings between $\mathrm{i}$-th and $(\mathrm{i}+1)$-th $\mathrm{D}$-branes. Therefore, we need to demand that the ordering of incoming states is noncommutative in the Feynman diagrams.

We consider the moduli space $\mathscr{F}\left(\widetilde{p}_{0, d} ; \widetilde{p}_{0,1}, \widetilde{p}_{1,2}, \ldots\right.$, $\left.\tilde{p}_{d-1, d}\right)$ of the Feynman diagrams that satisfy the above conditions, for incoming states $\tilde{\theta}_{n_{0,1}}\left[\tilde{p}_{0,1}\right], \widetilde{\theta}_{n_{1,2}}\left[\tilde{p}_{1,2}\right], \cdots$, $\widetilde{\theta}_{n_{d-1, d}}\left[\widetilde{p}_{d-1, d}\right]$, where $\widetilde{p}_{i-1, i}=\widetilde{m}_{i-1, i} / n_{i-1, i}=m_{i-1, i} / n_{i-1, i}-$ $\alpha_{i-1, i}\left(\alpha_{i-1, i} \in \mathbf{Z}, i=1, \ldots, d\right)$, and outgoing states $\tilde{\theta}_{n_{0, d}}\left[\tilde{p}_{0, d}\right]$, where $\tilde{p}_{0, d}=\widetilde{m}_{0, d} / n_{0, d}=\sum_{i=1}^{d} \widetilde{m}_{i-1, i} / n_{0, d}$. We also consider the zero- and one-dimensional subspaces of the moduli space: $\mathscr{F}_{0}\left(\widetilde{p}_{0, d} ; \widetilde{p}_{0,1}, \widetilde{p}_{1,2}, \ldots, \widetilde{p}_{d-1, d}\right)$ and $\mathscr{F}_{1}\left(\widetilde{p}_{0, d} ; \widetilde{p}_{0,1}, \widetilde{p}_{1,2}, \ldots, \widetilde{p}_{d-1, d}\right)$, respectively. Then, we obtain the following theorem.

Theorem 9 (correct Feynman diagram). If $\mathscr{F}_{0}\left(\widetilde{p}_{0, d} ; \widetilde{p}_{0,1}\right.$, $\left.\widetilde{p}_{1,2}, \ldots, \widetilde{p}_{d-1, d}\right) \neq \emptyset, \mathscr{F}_{0}\left(\widetilde{p}_{0, d} ; \widetilde{p}_{0,1}, \widetilde{p}_{1,2}, \ldots, \widetilde{p}_{d-1, d}\right)=\{\widetilde{\delta}\}$, that is, $\mathscr{F}_{0}\left(\widetilde{p}_{0, d} ; \widetilde{p}_{0,1}, \widetilde{p}_{1,2}, \ldots, \widetilde{p}_{d-1, d}\right)$, consists of only one element $\widetilde{\delta}$. Then, $\widetilde{\delta} \in \mathscr{F}_{0}\left(\widetilde{p}_{0, d} ; \widetilde{p}_{0,1}, \widetilde{p}_{1,2}, \ldots, \widetilde{p}_{d-1, d}\right)$ determines a correlation function $\left\langle\widetilde{p}_{0, d} ; \widetilde{p}_{0,1}, \widetilde{p}_{1,2}, \ldots, \widetilde{p}_{d-1, d}\right\rangle_{\widetilde{\delta}}$, which satisfies

$$
\begin{aligned}
& \sum_{\alpha_{1,2}, \ldots, \alpha_{d-1, d} \in \mathbf{Z}}\left\langle\widetilde{p}_{0, d} ; \widetilde{p}_{0,1}, \widetilde{p}_{1,2}, \ldots, \widetilde{p}_{d-1, d}\right\rangle_{\widetilde{\delta}} \theta_{n_{0, d}}\left[\widetilde{p}_{0, d}\right] \\
= & \theta_{n_{0,1}}\left[p_{0,1}\right] \theta_{n_{1,2}}\left[p_{1,2}\right] \cdots \theta_{n_{d-1, d}}\left[p_{d-1, d}\right] .
\end{aligned}
$$

Proof. First, we classify the Feynman diagrams. There is no internal vertex because $\operatorname{dim}\left(\mathscr{F}_{0}\right)=0$. Then, the vertices are external only: $\widetilde{p}_{0, d}, \widetilde{p}_{0,1}, \widetilde{p}_{1,2}, \ldots, \widetilde{p}_{d-1, d} \cdot \widetilde{p}_{0,1}$ is at the end of the diagrams because of the nearest neighbour interaction (Figure 2). $n_{0,1}>0$ because the string needs to propagate. 


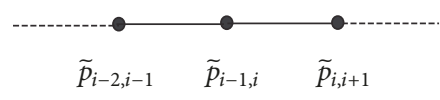

FIGURE 3: The external vertices that cannot propagate.

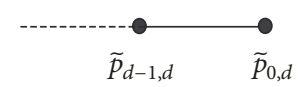

FIGURE 4: End point when $n_{0, d}<0$.

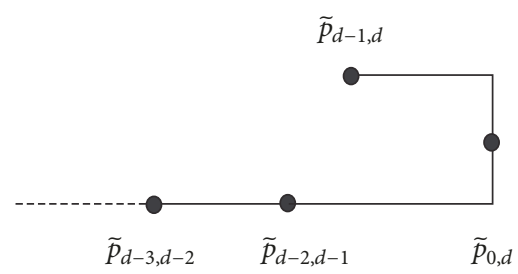

FIGURE 5: End point when $n_{0, d}>0$.

Because of the three point interactions, $\widetilde{p}_{i-1, i}$ not at the end of the diagrams cannot propagate, namely, $n_{i-1, i}<0$ (Figure 3 ). Let us consider the outgoing vertex $\widetilde{p}_{0, d}$. When $n_{0, d}<0$, a vertex propagate to $\widetilde{p}_{0, d}$ (Figure 4 ). When $n_{0, d}>0$, because no vertex propagates to $\widetilde{p}_{0, d}$, the last interaction must be as in (Figure 5). As a result, we obtain only (i) (Figure 6) when $n_{0, d}<0$ and (ii) (Figure 7) when $n_{0, d}>0$. Therefore, we have shown that $\mathscr{F}_{0}\left(\widetilde{p}_{0, d} ; \widetilde{p}_{0,1}, \widetilde{p}_{1,2}, \ldots, \widetilde{p}_{d-1, d}\right)$ consists of only one element $\widetilde{\delta}$ if $\mathscr{F}_{0}\left(\widetilde{p}_{0, d} ; \widetilde{p}_{0,1}, \widetilde{p}_{1,2}, \ldots, \widetilde{p}_{d-1, d}\right) \neq \emptyset$.

$\widetilde{\delta}$.

Next, we calculate the correlation function determined by

(i) When $n_{0, d}<0$, from the diagram in Figure 6,

$$
\begin{aligned}
& \sum_{\alpha_{1,2}, \ldots, \alpha_{d-1, d} \in \mathbf{Z}}\left\langle\tilde{p}_{0, d} ; \tilde{p}_{0,1}, \tilde{p}_{1,2}, \ldots, \tilde{p}_{d-1, d}\right\rangle_{\tilde{\delta}} \\
& \cdot \tilde{\theta}_{n_{0,1}+\cdots+n_{d-1, d}}\left[\tilde{p}_{0, d}\right] \\
& =\sum_{\alpha_{1,2}, \ldots, \alpha_{d-1, d} \in \mathbf{Z}} \widetilde{G}_{n_{0,1}}\left[\frac{\widetilde{m}_{0,1}}{n_{0,1}}, \frac{\widetilde{m}_{1,2}}{n_{1,2}}\right] \\
& \text {. } \widetilde{G}_{n_{0,1}+n_{1,2}}\left[\frac{\widetilde{m}_{1,2}}{n_{1,2}}, \frac{\widetilde{m}_{2,3}}{n_{2,3}}\right] \\
& \cdots \widetilde{G}_{n_{0,1}+n_{1,2}+\cdots+n_{d-2, d-1}}\left[\frac{\widetilde{m}_{d-2, d-1}}{n_{d-2, d-1}}, \frac{\widetilde{m}_{d-1, d}}{n_{d-1, d}}\right] \\
& \cdot \widetilde{G}_{n_{0,1}+n_{1,2}+\cdots+n_{d-1, d}}\left[\frac{\widetilde{m}_{d-1, d}}{n_{d-1, d}}, \frac{\widetilde{m}_{0, d}}{n_{0, d}}\right] \\
& \cdot \tilde{\theta}_{n_{0,1}+n_{1,2}+\cdots+n_{d-1, d}}\left[\frac{\widetilde{m}_{0, d}}{n_{0, d}}\right] \\
& =\sum_{\alpha_{1,2}, \ldots, \alpha_{d-1, d} \in \mathbf{Z}} \widetilde{G}_{n_{0,1}}\left[\frac{\widetilde{m}_{0,1}}{n_{0,1}}, \frac{\widetilde{m}_{1,2}}{n_{1,2}}\right] \\
& \cdot \widetilde{G}_{n_{0,1}+n_{1,2}}\left[\frac{\widetilde{m}_{1,2}}{n_{1,2}}, \frac{\widetilde{m}_{2,3}}{n_{2,3}}\right]
\end{aligned}
$$

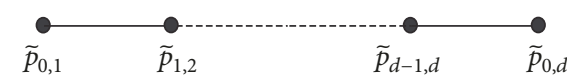

FiguRE 6: When $n_{0, d}<0$.

$$
\begin{aligned}
& \tilde{p}_{0,1} \tilde{p}_{1,2} \\
& \text { Figure 7: When } n_{0, d}>0 \text {. } \\
& \cdots \widetilde{G}_{n_{0,1}+n_{1,2}+\cdots+n_{d-2, d-1}}\left[\frac{\widetilde{m}_{d-2, d-1}}{n_{d-2, d-1}}, \frac{\widetilde{m}_{d-1, d}}{n_{d-1, d}}\right] \\
& \cdot \tilde{\theta}_{n_{0,1}+n_{1,2}+\cdots+n_{d-1, d}}\left[\frac{\widetilde{m}_{d-1, d}}{n_{d-1, d}}\right] \\
& =\sum_{\alpha_{1,2}, \ldots, \alpha_{d-1, d} \in \mathbf{Z}} \widetilde{G}_{n_{0,1}}\left[\frac{\widetilde{m}_{0,1}}{n_{0,1}}, \frac{\widetilde{m}_{1,2}}{n_{1,2}}\right] \\
& \cdot \widetilde{G}_{n_{0,1}+n_{1,2}}\left[\frac{\widetilde{m}_{1,2}}{n_{1,2}}, \frac{\widetilde{m}_{2,3}}{n_{2,3}}\right] \\
& \cdots \widetilde{G}_{n_{0,1}+n_{1,2}+\cdots+n_{d-2, d-1}}\left[\frac{\widetilde{m}_{d-2, d-1}}{n_{d-2, d-1}}, \frac{\widetilde{m}_{d-1, d}}{n_{d-1, d}}\right] \\
& \text { - } \widetilde{\theta}_{n_{0,1}+n_{1,2}+\cdots+n_{d-2, d-1}}\left[\frac{\widetilde{m}_{d-1, d}}{n_{d-1, d}}\right] \widetilde{\theta}_{n_{d-1, d}}\left[\frac{\widetilde{m}_{d-1, d}}{n_{d-1, d}}\right] \\
& =\sum_{\alpha_{1,2}, \ldots, \alpha_{d-1, d} \in \mathbf{Z}} \tilde{\theta}_{n_{0,1}}\left[\frac{\widetilde{m}_{0,1}}{n_{0,1}}\right] \tilde{\theta}_{n_{1,2}}\left[\frac{\widetilde{m}_{1,2}}{n_{1,2}}\right] \\
& \cdots \tilde{\theta}_{n_{d-1, d}}\left[\frac{\widetilde{m}_{d-1, d}}{n_{d-1, d}}\right] .
\end{aligned}
$$

From Lemma 6,

$$
\begin{aligned}
& \sum_{\alpha_{1,2}, \ldots, \alpha_{d-1, d} \in \mathbf{Z}}\left\langle\widetilde{p}_{0, d} ; \tilde{p}_{0,1}, \widetilde{p}_{1,2}, \ldots, \widetilde{p}_{d-1, d}\right\rangle_{\tilde{\delta}} \\
& \cdot \theta_{n_{0,1}+\cdots+n_{d-1, d}}\left[\widetilde{p}_{0, d}\right]=\theta_{n_{0,1}}\left[p_{0,1}\right] \theta_{n_{1,2}}\left[p_{1,2}\right] \\
& \cdots \theta_{n_{d-1, d}}\left[p_{d-1, d}\right] .
\end{aligned}
$$

(ii) When $n_{0, d}>0$, from the diagram in Figure 7 ,

$$
\begin{aligned}
& \sum_{\alpha_{1,2}, \ldots, \alpha_{d-1, d} \in \mathbf{Z}}\left\langle\tilde{p}_{0, d} ; \tilde{p}_{0,1}, \tilde{p}_{1,2}, \ldots, \widetilde{p}_{d-1, d}\right\rangle_{\tilde{\delta}} \\
& \cdot \widetilde{\theta}_{n_{0,1}+\cdots+n_{d-1, d}}\left[\widetilde{p}_{0, d}\right]
\end{aligned}
$$




$$
\begin{aligned}
& =\sum_{\alpha_{1,2}, \ldots, \alpha_{d-1, d} \in \mathbf{Z}} \widetilde{G}_{n_{0,1}}\left[\frac{\widetilde{m}_{0,1}}{n_{0,1}}, \frac{\widetilde{m}_{1,2}}{n_{1,2}}\right] \\
& \cdot \widetilde{G}_{n_{0,1}+n_{1,2}}\left[\frac{\widetilde{m}_{1,2}}{n_{1,2}}, \frac{\widetilde{m}_{2,3}}{n_{2,3}}\right] \\
& \cdots \widetilde{G}_{n_{0,1}+n_{1,2}+\cdots+n_{d-2, d-1}}\left[\frac{\widetilde{m}_{d-2, d-1}}{n_{d-2, d-1}}, \frac{\widetilde{m}_{0, d}}{n_{0, d}}\right] \\
& \cdot \widetilde{\theta}_{n_{0,1}+n_{1,2}+\cdots+n_{d-2, d-1}}\left[\frac{\widetilde{m}_{0, d}}{n_{0, d}}\right] \widetilde{G}_{n_{d-1, d}}\left[\frac{\widetilde{m}_{d-1, d}}{n_{d-1, d}}, \frac{\widetilde{m}_{0, d}}{n_{0, d}}\right] \\
& \cdot \widetilde{\theta}_{n_{d-1, d}}\left[\frac{\widetilde{m}_{0, d}}{n_{0, d}}\right] \\
& =\sum_{\alpha_{1,2}, \ldots, \alpha_{d-1, d} \in \mathbf{Z}} \tilde{\theta}_{n_{0,1}}\left[\frac{\widetilde{m}_{0,1}}{n_{0,1}}\right] \tilde{\theta}_{n_{1,2}}\left[\frac{\widetilde{m}_{1,2}}{n_{1,2}}\right] \\
& \cdots \widetilde{\theta}_{n_{d-2, d-1}}\left[\frac{\widetilde{m}_{d-2, d-1}}{n_{d-2, d-1}}\right] \widetilde{\theta}_{n_{d-1, d}}\left[\frac{\widetilde{m}_{d-1, d}}{n_{d-1, d}}\right] \text {. }
\end{aligned}
$$

From Lemma 6,

$$
\begin{aligned}
& \sum_{\alpha_{1,2}, \ldots, \alpha_{d-1, d} \in \mathbf{Z}}\left\langle\widetilde{p}_{0, d} ; \widetilde{p}_{0,1}, \widetilde{p}_{1,2}, \ldots, \widetilde{p}_{d-1, d}\right\rangle_{\widetilde{\delta}} \\
& \cdot \theta_{n_{0,1}+\cdots+n_{d-1, d}}\left[\tilde{p}_{0, d}\right]=\theta_{n_{0,1}}\left[p_{0,1}\right] \theta_{n_{1,2}}\left[p_{1,2}\right] \\
& \cdots \theta_{n_{d-1, d}}\left[p_{d-1, d}\right] .
\end{aligned}
$$

By using these Feynman diagrams, we define $m_{d}$.

Definition $10\left(m_{d}\right)$.

$$
\begin{aligned}
m_{d} & \left(p_{0,1}, p_{1,2}, \ldots, p_{d-1, d}\right) \\
& :=\sum_{\alpha_{1,2}, \ldots, \alpha_{d-1, d} \in \mathbf{Z}} \sum_{\tilde{\delta} \in \mathscr{F}_{0}\left(\widetilde{p}_{0, d} ; \widetilde{p}_{0,1}, \widetilde{p}_{1,2}, \ldots, \widetilde{p}_{d-1, d}\right)}(-1)^{|\widetilde{\delta}|} \\
& \cdot\left\langle\widetilde{p}_{0, d} ; \tilde{p}_{0,1}, \widetilde{p}_{1,2}, \ldots, \tilde{p}_{d-1, d}\right\rangle_{\widetilde{\delta}} \theta_{n_{0, d}}\left[\widetilde{p}_{0, d}\right]
\end{aligned}
$$

$|\widetilde{\delta}| \in \mathbf{Z}$ should be determined so that $m_{d}$ satisfy the $A_{\infty}$ relation. We have given a general definition including more than one-dimensional case, whereas $\widetilde{\delta}$ is unique in this one-dimensional situation. Although $m_{d}$ are essentially products of the theta functions as one can see in Theorem 9, the naturally defined directions of the propagators and noncommutativity of the external vertices restrict the states of the external vertices. As a result, we obtain the following theorems.

Theorem 11. The degree of $m_{d}$ is 2-d.

Proof. Because $p$ in $H^{0, p}\left(E_{\tau}, L\left(n_{i-1, i}, u_{i-1, i}\right)\right)$ represent the degrees of the open string states, the states with $n_{i-1, i}>0$ and $n_{i-1, i}<0$ have degrees 0 and 1 , respectively. In the proof of Theorem 9, the Feynman diagrams are classified into two kinds (Figures 6 and 7). In the diagrams in Figure 6, $n_{0, d}<0$, $n_{0,1}>0$, and $n_{i-1, i}<0(i=2, \cdots d)$. If we compare the degrees of the both sides of the formula in Definition 10, we obtain $0+(d-1)+\left(\right.$ the degree of $\left.m_{d}\right)=1$. Thus, the degree of $m_{d}$ is $2-d$. Because $n_{0, d}>0, n_{0,1}>0, n_{i-1, i}<0$ $(i=2, \cdots d-1)$, and $n_{d-1, d}>0$ in the diagrams in Figure 7, $0+(d-2)+0+\left(\right.$ the degree of $\left.m_{d}\right)=0$. Thus, the degree of $m_{d}$ is $2-d$.

Theorem $12\left(A_{\infty}\right.$ relation).

$$
\begin{aligned}
& \sum_{1 \leq p \leq d, 0 \leq q \leq d-p}(-1)^{\operatorname{deg}\left(p_{0,1}\right)+\cdots+\operatorname{deg}\left(p_{q-1, q}\right)-q} m_{d-p+1}\left(p_{0,1},\right. \\
& \ldots, p_{q-1, q}, m_{p}\left(p_{q, q+1}, \ldots, p_{p+q-1, p+q}\right), p_{p+q, p+q+1}, \ldots, \\
& \left.p_{d-1, d}\right)=0,
\end{aligned}
$$

where $\operatorname{deg}\left(p_{i-1, i}\right)(i=1, \ldots, d)$ represent the degrees of the string states.

Proof.

$$
\begin{aligned}
& m_{d-p+1}\left(p_{0,1}, \ldots, p_{q-1, q}, m_{p}\left(p_{q, q+1}, \ldots, p_{p+q-1, p+q}\right),\right. \\
& \left.p_{p+q, p+q+1}, \ldots, p_{d-1, d}\right) \\
& =\sum_{\alpha_{q+1, q+2}, \ldots, \alpha_{p+q-1, p+q} \in \mathbf{Z}} \sum_{\widetilde{\delta}_{1} \in \mathscr{F}_{0}\left(\widetilde{r} ; \tilde{p}_{q, q+1}, \ldots, \widetilde{p}_{p+q-1, p+q}\right)}(-1)^{\mid \widetilde{\delta_{1} \mid}}\left\langle\widetilde{r} ; \widetilde{p}_{q, q+1}, \ldots, \widetilde{p}_{p+q-1, p+q}\right\rangle_{\widetilde{\delta}_{1}} \\
& m_{d-p+1}\left(p_{0,1}, \ldots, p_{q-1, q}, \widetilde{r}, p_{p+q, p+q+1}, \ldots, p_{d-1, d}\right) \\
& =\sum_{\alpha_{0,1}, \ldots, \alpha_{q-1, q}, \alpha_{q+1, q+2}, \ldots, \alpha_{d-1, d} \in \mathbf{Z}} \sum_{\tilde{\delta}_{1} \in \mathscr{F}_{0}\left(\widetilde{r} ; \widetilde{p}_{q, q+1}, \ldots, \widetilde{p}_{p+q-1, p+q}\right)} \sum_{\tilde{\delta}_{2} \in \mathscr{F}_{0}\left(\widetilde{p}_{0, d} ; \tilde{p}_{0,1}, \ldots, \tilde{p}_{q-1, q}, \tilde{r}, \widetilde{p}_{p+q, p+q+1}, \ldots, \tilde{p}_{d-1, d}\right)}(-1)^{\left|\widetilde{\delta_{1}}\right|+\left|\widetilde{\delta_{1}}\right|}\left\langle\widetilde{r} ; \widetilde{p}_{q, q+1}, \ldots, \widetilde{p}_{p+q-1, p+q}\right\rangle_{\tilde{\delta}_{1}} \\
& \left\langle\widetilde{p}_{0, d} ; \tilde{p}_{0,1}, \ldots, \widetilde{p}_{q-1, q}, \widetilde{r}, \tilde{p}_{p+q, p+q+1}, \ldots, \widetilde{p}_{d-1, d}\right\rangle_{\widetilde{\delta}_{2}} \theta_{n_{0,1}+\cdots+n_{d-1, d}}\left[\tilde{p}_{0, d}\right] .
\end{aligned}
$$



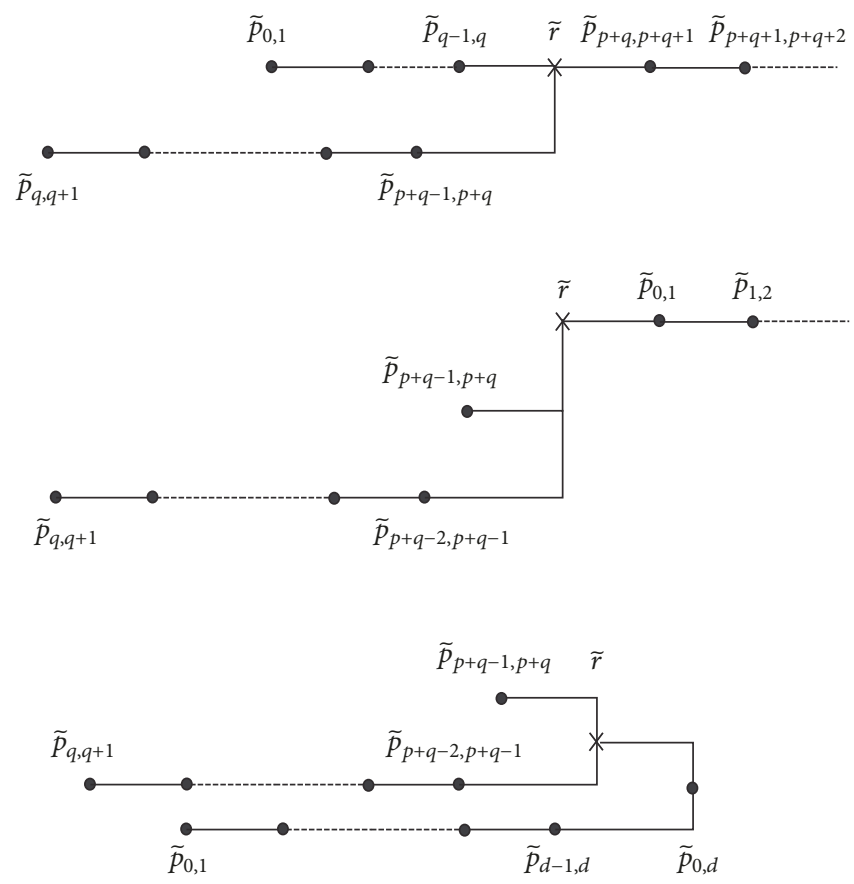

FIGURE 8: $\widetilde{\delta}_{1} \otimes \widetilde{\delta}_{2} \in \partial \overline{\mathscr{F}}_{1}$.

When $\tilde{r}$ take arbitrary values, the diagrams in Figure 8 are elements of $\overline{\mathscr{F}}_{1}\left(\widetilde{p}_{0, d} ; \widetilde{p}_{0,1}, \widetilde{p}_{1,2}, \ldots, \widetilde{p}_{d-1, d}\right)$, which is the onedimensional subspace of $\overline{\mathscr{F}}\left(\tilde{p}_{0, d} ; \tilde{p}_{0,1}, \widetilde{p}_{1,2}, \ldots, \tilde{p}_{d-1, d}\right)$, where $\overline{\mathscr{F}}\left(\widetilde{p}_{0, d} ; \widetilde{p}_{0,1}, \widetilde{p}_{1,2}, \ldots, \widetilde{p}_{d-1, d}\right)$ is a closure of $\mathscr{F}\left(\widetilde{p}_{0, d} ; \widetilde{p}_{0,1}, \widetilde{p}_{1,2}, \ldots, \widetilde{p}_{d-1, d}\right)$. Especially, $\tilde{\delta}_{1} \otimes \tilde{\delta}_{2}$ are the diagrams in Figure 8 with $\widetilde{r}=\left(n_{q, q+1} \widetilde{p}_{q, q+1}+\right.$ $\left.\cdots+n_{q+p-1, q+p} \tilde{p}_{q+p-1, q+p}\right) /\left(n_{q, q+1}+\cdots+n_{q+p-1, q+p}\right)$, which are elements of $\partial \widetilde{\mathscr{F}}_{1}\left(\widetilde{p}_{0, d} ; \widetilde{p}_{0,1}, \widetilde{p}_{1,2}, \ldots, \widetilde{p}_{d-1, d}\right)$, where $\partial \overline{\mathscr{F}}_{1}\left(\widetilde{p}_{0, d} ; \tilde{p}_{0,1}, \widetilde{p}_{1,2}, \ldots, \widetilde{p}_{d-1, d}\right)$ is a boundary of $\overline{\mathscr{F}}_{1}\left(\tilde{p}_{0, d} ; \tilde{p}_{0,1}, \widetilde{p}_{1,2}, \ldots, \widetilde{p}_{d-1, d}\right)$. By choosing $|\widetilde{\delta}|$ appropriately, (40) becomes 0 because the contributions from the boundary of the one-dimensional space cancel with each other as in the same mechanism in the A-model $[6,9,22]$.

Here we compare $m_{d}$ in the B-model and A-model.

Theorem 13 (mirror symmetry). $m_{d}$ in the B-model in Definition 10 coincides with $m_{d}$ in the A-model by identifying $\theta_{n}[\widetilde{p}]$ with $[\tilde{p}]$ (This identification is a quasi-isomorphism [6, 9].).

Proof. The formula in Definition 10 can be regarded as $m_{d}$ in the A-model by replacing $\theta_{n}[\tilde{p}]$ with $[\tilde{p}]$. The following is the definition of the formula in Definition 10 in the Amodel $[6,9]$. In the A-model, the $\mathrm{i}$-th D-brane (Lagrangian submanifold) $\widetilde{L}_{i}(i=1, \ldots, d)$ is represented by a line with slope $-n_{i}$ on the universal cover of the symplectic torus TB. The intersection of $\widetilde{L}_{i-1}$ and $\widetilde{L}_{i}$ is represented by $\tilde{y}=\widetilde{p}_{i-1, i}$ in $(\tilde{y}, \tilde{x})$ coordinates that is the universal cover of $(y, x)$ coordinates of $T B(0 \leq y, x<1)$. $\left[\widetilde{p}_{i-1, i}\right]$ represents the string state with $n_{i-1, i}$ between the D-branes $\widetilde{L}_{i-1}$ and $\widetilde{L}_{i}$. [ $\left.\tilde{p}_{i-1, i}\right]$ corresponds to $\theta_{n_{i-1, i}}\left[\widetilde{p}_{i-1, i}\right]$ in the B-model. The intersection of $L_{d}$ and $L_{0}$ is determined as $\widetilde{p}_{0, d}=\left(1 / n_{0, d}\right) \sum_{i=1}^{d} n_{i-1, i} \widetilde{p}_{i-1, i}$. $\left[\tilde{p}_{0, d}\right]$ is a basis of the $m_{d}\left(p_{0,1}, p_{1,2}, \ldots, p_{d-1, d}\right)$, which corresponds to $\theta_{n_{0, d}}\left[\widetilde{p}_{0, d}\right]$ in the $\mathrm{B}$-model. The area of the convex surrounded by $L_{0}, \ldots, L_{d}$ is determined as $S_{d}\left(\tilde{p}_{0,1}, \ldots, \widetilde{p}_{d-1, d}\right)=(1 / 2)\left(\sum_{i=1}^{d} n_{i-1, i} \tilde{p}_{i-1, i}\left(\tilde{p}_{i-1, i}-1\right)-\right.$ $\left.n_{0, d} \widetilde{p}_{0, d}\left(\widetilde{p}_{0, d}-1\right)\right) \cdot \exp \left(2 \pi i \tau S_{d}\left(\widetilde{p}_{0,1}, \ldots, \widetilde{p}_{d-1, d}\right)\right)$ is the coefficient of the $m_{d}\left(p_{0,1}, p_{1,2}, \ldots, p_{d-1, d}\right)$, which is equal to the coefficient of the $m_{d}\left(p_{0,1}, p_{1,2}, \ldots, p_{d-1, d}\right)$ in the B-model as one can see in Theorems 4 and 9 .

We are going to show that the Feynman diagrams, which determine the $A_{\infty}$ structure of the B-model, coincide with the tropical Morse trees, which determine the $A_{\infty}$ structure of the A-model. That is, both the $A_{\infty}$ structures coincide. The tropical Morse trees $\widetilde{\phi}$ are continuous maps that satisfy the following conditions, (The condition (6) in [9] determines not the tropical Morse trees but the length of the metric ribbon trees from the tropical Morse trees.) from the metric ribbon trees $S$ to the universal cover $B(\ni \tilde{y})$ of $\mathbf{R} / \mathbf{Z}(\ni y)[6,9]$.

(0) We attach $n_{i-1, i}$ to the i-th external incoming edge and attach the sum of all numbers labelling the edges coming into a vertex to the edge coming out of the vertex. That is, the numbers $n$ are preserved at vertices. We define an affine displacement vector $v$ on each edge.

(1) The coordinates of the external incoming and outgoing vertices are $\tilde{y}=\widetilde{p}_{i-1, i}$ and $\widetilde{y}=\widetilde{p}_{0, d}$, respectively.

(2) All the edges and vertices of the metric ribbon trees map to the edges and vertices in the universal cover, respectively.

(3) $v=0$ at the external vertices. 
(4) $\Delta v=n \Delta \tilde{y}$ on the edges. The directions of $v$ and the edges coincide.

(5) $v$ are preserved at the vertices.

The conditions $(0), \ldots,(5)$ coincide with the properties of the Feynman diagrams in the B-model: (1) is satisfied in the B-model by Theorem 9. (2) is automatically satisfied in the B-model. (0), (3), and (5) follow from the definition of the canonical form of the $\widetilde{\theta}$. (4) coincides with the condition that $\Delta v=n \Delta \widetilde{p}$ and directions of the propagators are the same as those of $v$.

Because the Feynman diagrams in the B-model coincide with the tropical Morse trees in the A-model, $\mathscr{F}\left(p_{0, d}\right.$; $\left.p_{0,1}, p_{1,2}, \ldots, p_{d-1, d}\right)=S^{\text {trop }}\left(p_{0, d} ; p_{0,1}, p_{1,2}, \ldots, p_{d-1, d}\right)$, which is the moduli space of the tropical Morse trees. It is shown in $[6,9]$ that $S^{\text {trop }}\left(p_{0, d} ; p_{0,1}, p_{1,2}, \ldots, p_{d-1, d}\right)$ is bijective (The bijection map is given as follows $[6,9]$ : if the tropical Morse trees are decomposed to the edges, the corresponding pseudo holomorphic curves are also decomposed and, thus, the moduli spaces are decomposed as $\delta^{\text {trop }}=\bigcup_{e} \delta_{e}^{\text {trop }}$ and $\mathscr{M} / P S L(2, \mathbf{R})=\bigcup_{e} \mathscr{M}_{e} / P S L(2, \mathbf{R})$. If the decomposed tropical Morse trees are denoted as $\mathcal{S}_{e}^{\text {trop }} \ni \tilde{\phi}: S_{e} \longrightarrow$ $B$ defined by $s \longmapsto \widetilde{\phi}(s)(0 \leq s \leq 1)$, the decomposed pseudo holomorphic curves are denoted as $\mathscr{M}_{e} / P S L(2, \mathbf{R}) \ni$ $R_{\tilde{\phi}}: D_{e} \longrightarrow T B$ defined by $(s, t) \longmapsto\left(\widetilde{\phi}(s),-n_{e} \widetilde{\phi}(s)-\right.$ $t(d / d s) \tilde{\phi}(s))(0 \leq t \leq 1)$, where $(d / d s) \tilde{\phi}(s)=v(s)$. Then, the map between these moduli spaces is given by $F: \mathcal{S}_{e}^{\text {trop }} \longrightarrow \mathscr{M}_{e} / \operatorname{PSL}(2, \mathbf{R})$ defined by $\tilde{\phi} \longmapsto R_{\tilde{\phi}}$, that is, $\widetilde{\phi}(s) \longmapsto\left(\widetilde{\phi}(s),-n_{e} \widetilde{\phi}(s)-t(d / d s) \widetilde{\phi}(s)\right)$.) to the moduli space $\mathscr{M}\left(p_{0, d} ; p_{0,1}, p_{1,2}, \ldots, p_{d-1, d}\right) / \operatorname{PSL}(2, \mathbf{R})$ of the pseudo holomorphic maps from a disk with $d+1$ marked points to the symplectic torus $T B$ in the A-model in one dimension. If a topology, for example Gromov topology [23], is defined on $\mathscr{M}\left(p_{0, d} ; p_{0,1}, p_{1,2}, \ldots, p_{d-1, d}\right) / \operatorname{PSL}(2, \mathbf{R})$, a topology is uniquely induced on $S^{\text {trop }}\left(p_{0, d} ; p_{0,1}, p_{1,2}, \ldots, p_{d-1, d}\right)$ by the bijective map (For example, if $\mathscr{M}_{e} / \operatorname{PSL}(2, \mathbf{R})$ is equipped with the topology of the functional space, equivalently, compact-open topology, the same topology is induced to $\mathcal{S}_{e}^{\text {trop }}$ and $\mathcal{S}_{e}^{\text {trop }} \cong \mathscr{M}_{e} / P S L(2, \mathbf{R})$. By patching them together, $\mathcal{S}^{\text {trop }} \cong \mathscr{M} / P S L(2, \mathbf{R})$.). As a result, we obtain the following.

Theorem 14 (topological space in homological mirror symmetry). $\mathscr{F}\left(p_{0, d} ; p_{0,1}, p_{1,2}, \ldots, p_{d-1, d}\right)$ in the B-model is homeomorphic to $\mathscr{M}\left(p_{0, d} ; p_{0,1}, p_{1,2}, \ldots, p_{d-1, d}\right) / \operatorname{PSL}(2, \mathbf{R})$ in the $A$ model.

\section{Conclusion and Discussion}

In this paper, we have defined and explicitly constructed $m_{d}$ that form $A_{\infty}$-category of the B-model on an elliptic curve. The way to define $m_{d}$ is summarized as follows. The morphisms in the DG-category of this model are represented by theta functions with characteristics. From the products of the two theta functions, we can derive Feynman rules in the space of the characteristics, in other words, the configuration space of the morphisms. $m_{d}$ are defined by the products of $d$ theta functions with the characteristics in the case that the moduli space of the Feynman diagrams is restricted to zerodimensional. This restriction is necessary for $m_{d}$ to satisfy the $A_{\infty}$ relations. This $m_{d}$ algebra coincides with the $m_{d}$ algebra in the Fukaya category of the A-model on the corresponding symplectic torus. Thus, the $A_{\infty}$-category formed by these $m_{d}$ is equivalent to the Fukaya category as an $A_{\infty}$-category. We have also shown that the moduli space of the Feynman diagrams in the B-model is homeomorphic to the moduli space of the pseudo holomorphic curves in the A-model.

The way to define $m_{d}$ is naturally generalized in the case of the B-model on $2 n$-dimensional complex manifolds: we derive Feynman rules in the configuration space of the morphisms from the products of the two morphisms in the DG-category of the B-model. Then we define $m_{d}$ by the products of $d$ morphisms with the configurations in the case that the moduli space of the Feynman diagrams is restricted to zero-dimensional. We conjecture that these $m_{d}$ satisfy the $A_{\infty}$ relations and form an $A_{\infty}$-category, which is equivalent to the Fukaya category of the A-model on the corresponding $2 n$-dimensional symplectic manifold, as an $A_{\infty}$-category. We also conjecture that the moduli space of the Feynman diagrams in the B-model on the $2 n$-dimensional complex manifolds is homeomorphic to the moduli space of the pseudo holomorphic curves in the A-model on the corresponding $2 n$-dimensional symplectic manifold. These moduli spaces determine the $A_{\infty}$ structure of the both models.

\section{Data Availability}

No data were used to support this study.

\section{Conflicts of Interest}

The author declares that they have no conflicts of interest.

\section{References}

[1] M. Kontsevich, "Homological algebra of mirror symmetry," in the International Congress of Mathematicians, pp. 120-139, Birkhäuser, Basel, Switzerland.

[2] A. Polishchuk and E. Zaslow, "Categorical mirror symmetry: the elliptic curve," Advances in Theoretical and Mathematical Physics, vol. 2, no. 2, pp. 443-470, 1998.

[3] K. Fukaya and Y.-G. Oh, "Zero-loop open strings in the cotangent bundle and Morse homotopy," Asian Journal of Mathematics, vol. 1, no. 1, pp. 96-180, 1997.

[4] M. Kontsevich and Y. Soibelman, "Homological mirror symmetry and torus fibrations," in Symplectic geometry and mirror symmetry (Seoul, 2000), pp. 203-263, World Sci. Publ., River Edge, NJ, 2001.

[5] M. Abouzaid, "Morse homology, tropical geometry, and homological mirror symmetry for toric varieties," Selecta Mathematica-New Series, vol. 15, no. 2, pp. 189-270, 2009.

[6] M. Abouzaid, On homological mirror symmetry for toric varieties, University of Chicago, 2007.

[7] M. Gross, Tropical geometry and mirror symmetry, vol. 114 of CBMS Regional Conference Series in Mathematics, Published for 
the Conference Board of the Mathematical Sciences, Washington, DC; by the American Mathematical Society, Providence, RI, 2011.

[8] M. Gross and B. Siebert, "Theta functions and mirror symmetry," Algebraic Geometry (math.AG), vol. 21, pp. 95-138, 2016.

[9] P. S. Aspinwall, T. Bridgeland, A. Craw et al., Dirichlet branes and mirror symmetry, vol. 4, American Mathematical Society, Providence, RI, USA, 2009.

[10] E. Witten, "Chern-Simons gauge theory as a string theory," Mathematical Programming, vol. 133, pp. 637-678, 1995.

[11] S. A. Merkulov, "Strongly homotopy algebras of a Kahler manifold," International Mathematics Research Notices, vol. 3, Article ID 9809172, p. 153, 1999.

[12] A. Polishchuk, "Massey and Fukaya products on elliptic curves," Advances in Theoretical and Mathematical Physics, vol. 4, no. 6, pp. 1187-1207, 2000.

[13] A. Polishchuk, "Homological mirror symmetry with higher products," Mathematics, Algebraic Geometry, vol. 1, 1999.

[14] A. Polishchuk, " $\mathrm{A}_{\infty}$-structures on an elliptic curve," Communications in Mathematical Physics, vol. 247, no. 3, pp. 527-551, 2004.

[15] P. S. Aspinwall and S. Katz, "Computation of superpotentials for D-branes," Communications in Mathematical Physics, vol. 264, no. 1, pp. 227-253, 2006.

[16] E. Zaslow, "Seidel's mirror map for the torus," Advances in Theoretical and Mathematical Physics, vol. 9, no. 6, pp. 999-1006, 2005.

[17] K. Kobayashi, "On exact triangles consisting of stable vector bundles on tori," Differential Geometry and its Applications, vol. 53, pp. 268-292, 2017.

[18] A. Kanazawa, "Doran-Harder-Thompson conjecture via SYZ mirror symmetry: elliptic curves," SIGMA, vol. 13, Paper No. 024, 13 pages, 2017.

[19] M. F. Atiyah, "Vector bundles over an elliptic curve," Proceedings of the London Mathematical Society. Third Series, vol. 7, pp. 414452, 1957.

[20] E. Witten, "Mirror manifolds and topological field theory," in Mirror Symmetry I, S. T. Yau, Ed., vol. 9, pp. 121-160, 1998.

[21] D. Mumford, Tata Lectures on Theta I, vol. 28 of Progress in Mathematics, Birkhäuser Boston, Inc., Boston, MA, USA, 1983.

[22] P. Seidel, Fukaya Categories and Picard-Lefschetz Theory, European Mathematical Society, 2008.

[23] M. Gromov, "Pseudo holomorphic curves in symplectic manifolds," Inventiones Mathematicae, vol. 82, no. 2, pp. 307-347, 1985. 


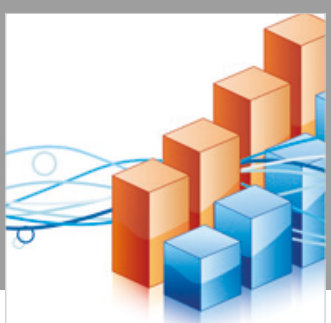

Advances in

Operations Research

\section{-n-m}
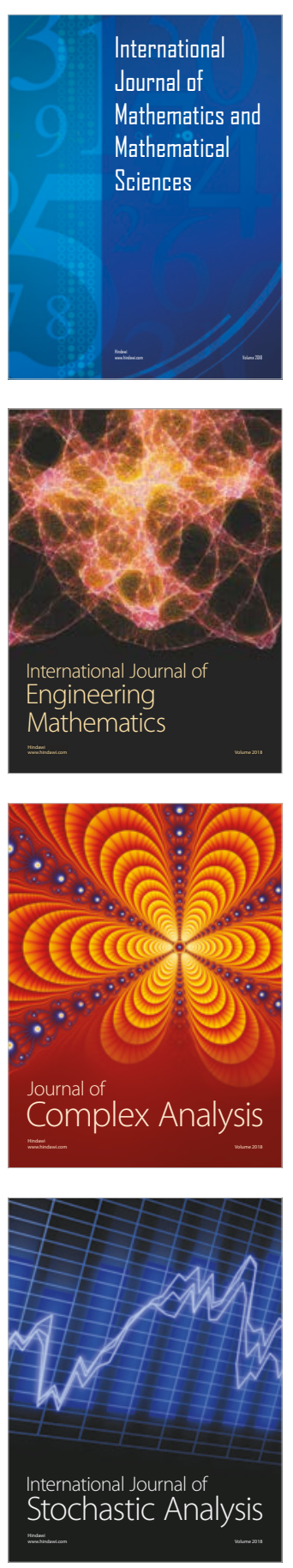
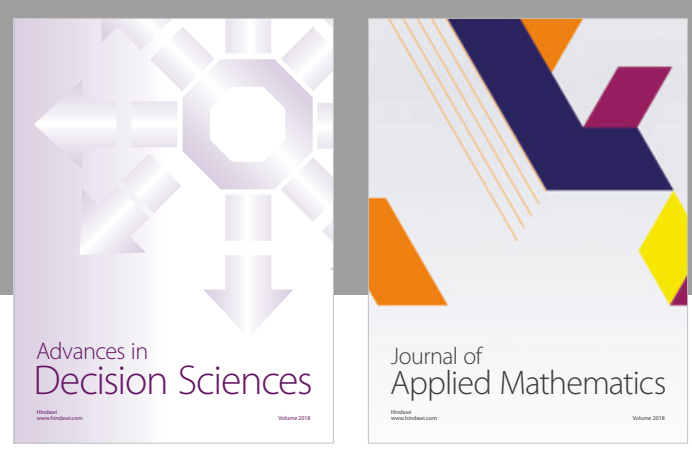

Journal of

Applied Mathematics
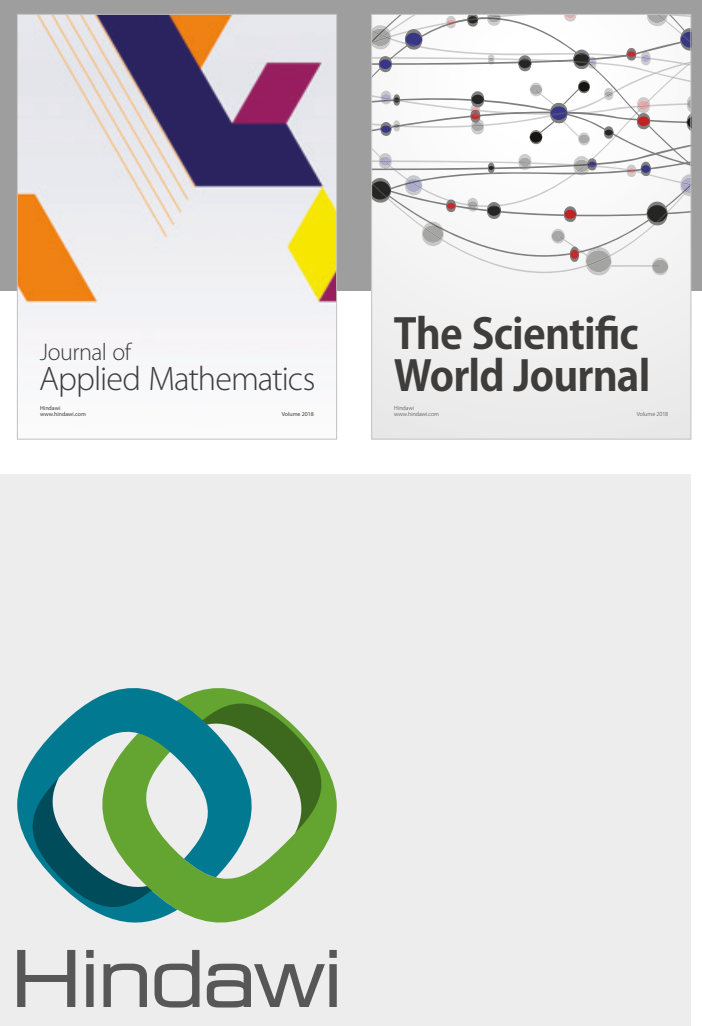

Submit your manuscripts at

www.hindawi.com

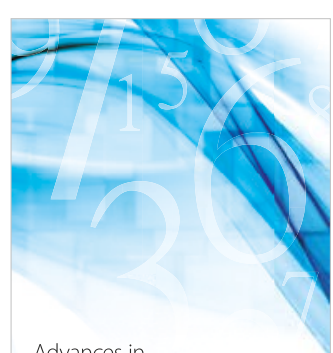

Advances in
Numerical Analysis
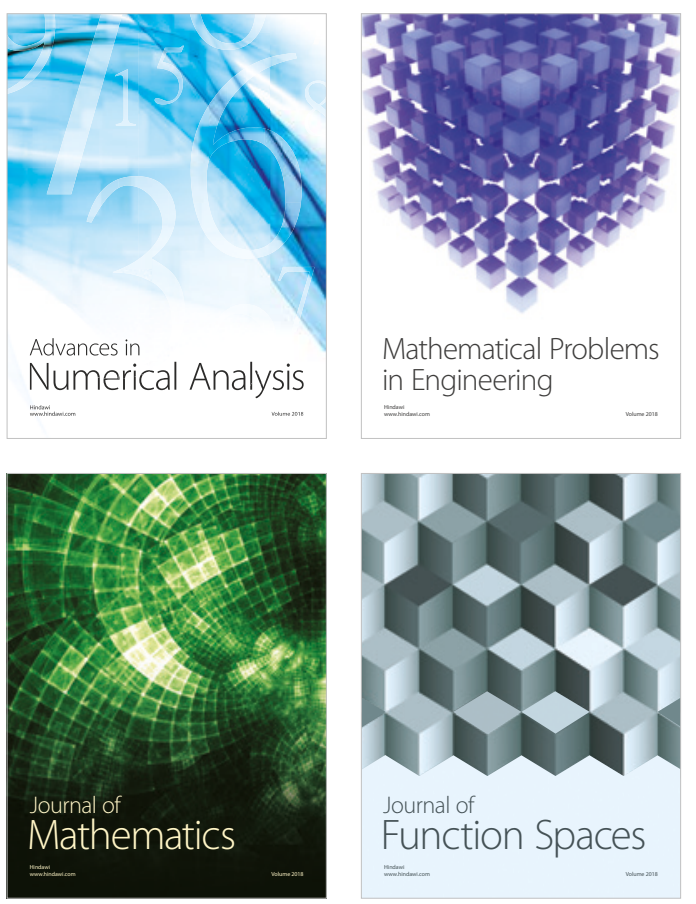

Mathematical Problems in Engineering

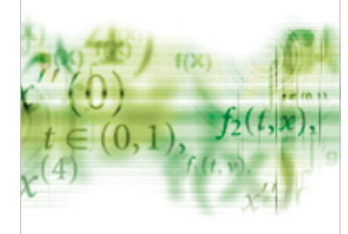

International Journal of

Differential Equations

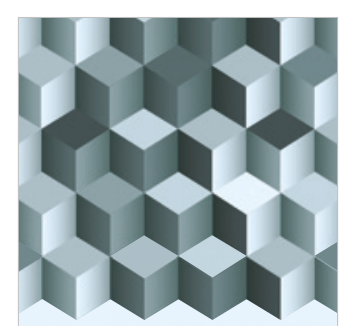

Journal of

Function Spaces

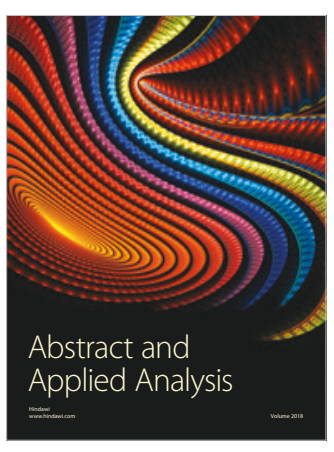

The Scientific

World Journal

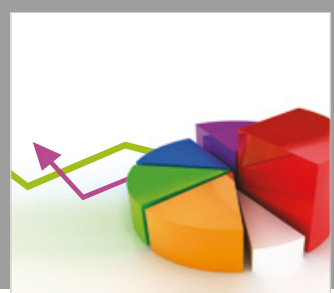

Journal of

Probability and Statistics
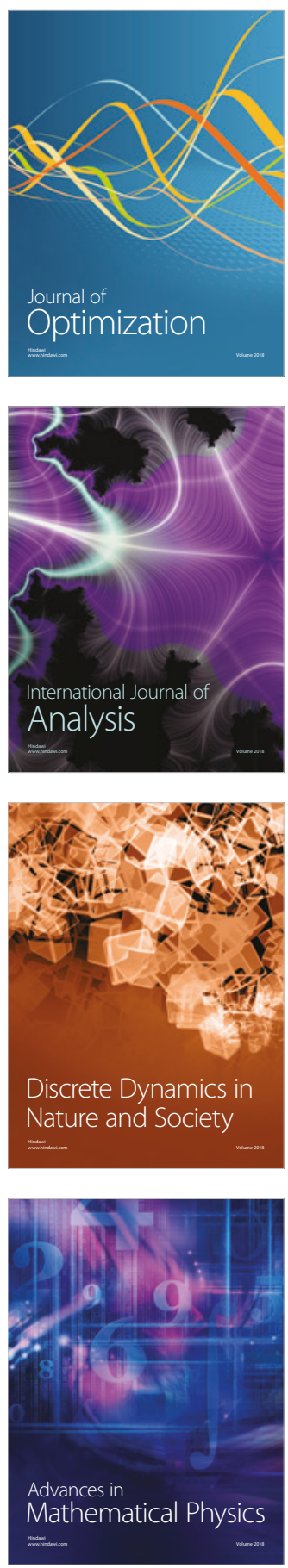